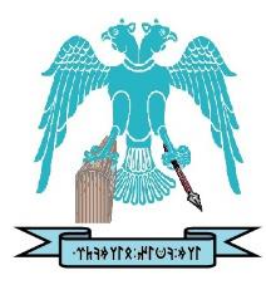

JOURNAL OF ENERGY SYSTEMS

$2019,3(4)$

\title{
Experimental realization of electromigration at high power for copper wires
}

\author{
Ibrahim Mahariq \\ American University of the Middle East, College of Engineering and Technology, Kuwait, Kuwait, \\ ibrahim.maharik@aum.edu.kw \\ Svetlana Berjozkina iD \\ American University of the Middle East, College of Engineering and Technology, Kuwait, Kuwait, \\ svetlana.berjozkina@aum.edu.kw
}

\begin{tabular}{rr|r|} 
Submitted: & 08.09.2019 \\
Accepted: & 09.12.2019 \\
Published: & 31.12 .2019 & $\begin{array}{c}\text { Check for } \\
\text { updates }\end{array}$ \\
\hline
\end{tabular}

\begin{abstract}
The electromigration phenomenon is a topical concern in all types of interconnects in power systems for both low and high voltages, low and large power ranges, for instance, in fabrication and manufacturing of Integrated Circuits, electronics of the power modules, power grid interconnects etc. Due to distributed generation units, in particular, renewable energy sources, which are commonly connected to already existing infrastructures, the considered issues related to the electromigration impact will result to a potential reduction of the electrical interconnection failure risks. Thus, the design of the integrated systems has to perform at highly efficient level with low probability of disconnections. The presented study considers the effect of the electromigration in terms of the spatial direction of the currents by observing directions of the sparks under certain arrangements of copper wires' connections. The experimental setup has been developed in order to realize and observe the theoretical expectations. The experimental results were conducted at around $500 \mathrm{~A}$, and the corresponding observations match theoretical expectations.
\end{abstract}

Keywords: Electromigration, High power, Interconnect reliability, Physical design

Cite this paper as: $\quad$ Mahariq, I., Berjozkina, S., Experimental realization of electromigration at high power for copper wires. Journal of Energy Systems 2019, 3(4), 158-167, DOI: 10.30521/jes. 616982

(c) 2019 Published by peer-reviewed open access scientific journal, JES at DergiPark (https://dergipark.org.tr/en/pub/jes) 


\section{INTRODUCTION}

The electromigration (EM) phenomenon [1] (material migration caused by an electric field) has been discovered for over one hundred years by French Scientist M. Gerardin [2]. The EM is one of the failure reasons for Integrated Circuits (ICs) interconnects since the first packaged ICs have been appeared in the late 1960s. In fact, ICs and semiconductor chips play a considerable role in the applications of modern power electronics nowadays, for instance, in a computer controlled and operated machinery, telecommunication, mechatronics, and etc.

In order to ensure a high-efficient and low-cost design of this technology, numerous required features that provide inhomogeneities have to be considered in the wiring of the packaged IC chip is performed, for example, current densities and temperature distributions are varying, material changes, manufacturing issues related to the tolerances as well as some existing damages, etc [3]. As a result, the above-mentioned features impact the IC chip design leading to damage interconnect (hillocks that cause short circuits). Moreover, EM causes whisker growth phenomenon in wires [4] that results in shortcircuit and arcing in electronic circuits.

Since reliability of electronic systems (including ICs and chips) is a core issue for implementators, the new factors and effects have to be considered, in particular, related to EM phenomenon in order to improve the reliability of these systems [3].

Wide-ranging studies on EM have been performed in the past and going on currently by modeling, observing and discovering wire bonding degradation in microelectronics. Several theoretical and experimental justifications were revealed in order to ensure better understanding of EM phenomenon and finding ways to predict, for instance, its' weak spots and time to failure, as well as to access the reliability of the IC components. The biggest part of previous studies has been performed by testing structures, for instance, useful for the evaluation of interconnects in water fabrication, other part - at the circuit layout level. Let's examine contribution of the previous works.

The consideration of the failure mechanism in the heel region for connections of power electronic modules through investigation of the process impact on the connection's reliability is shown in [5]. The Finite Element Method (FEM) approach was applied for the forming process of the ribbon. The authors proposed an approach based on the critical dissipated energy of the material for the ribbon life time prediction taking into account the electrical load.

The study related to power electronics reliability by considering the load and the degradation mechanisms due to thermal cycling in terms of the material behavior shown in [6], where the author considers thermal-mechanical models. The obtained results for the bond-wire lift-off failures and for crack propagation in large-area solder joints are presented.

Since the microelectronic packages include such metals as aluminum, copper, nickel, gold and silver, the Intermetallic Compound (IMC) should be considered too because of the metal contact. Excessive IMC growth is one of the reasons for bonding failure since IMC varies under different conditions. The study related to the examination of the effect of moisture and temperature on $\mathrm{Al}-\mathrm{Cu}$ interfacial strength is presented in [7], where authors investigate IMC growth and interfacial adhesion of $\mathrm{Al}$ wire bond on $\mathrm{Cu}$ based lead frame with Bare $\mathrm{Cu}$ surface, Ni plated surface and Ag plated surface. The study related to the reliability of $\mathrm{Cu} / \mathrm{Al}$ bond in comparison with that of $\mathrm{Au} / \mathrm{Al}$ bond at comparable temperature is presented in $[8,9]$. Research related to the effects of Ni nanoparticles addition on EM under high current 
density was studied in [10], where authors presented reduction approach of the EM damage in SAC305 (lead free solder SN96) solder joints in order to ensure more reliable solder joints.

One of the studies related to the solder joint behavior in HCC/PWB interconnections was performed and observed in [11], where authors demonstrated analytical modeling and experimental results. The authors claim that considerable factors such as the solder joint composition and microstructure impact the overall reliability of HCC/PWB interconnect in terms of the thermal stability of the solder.

The reliability of the thermal fatigue fracture of the solder joint for power cycle in power module was analyzed in [12 - 14], where both the thermal fatigue life of a power module and factors affecting it are examined. FEM has been used in order to establish improved evaluation for the thermal fatigue life of power module. The coupled electrical-thermal-structural analysis has been applied by precisely evaluating temperature distribution inside the power module (Joule heating effect). The consideration included the solder thickness, the void ratio, the differences in crack mode and the real thermal boundary conditions.

The adopted physics-of-failure-based life prediction models for wire bond interconnects in power electronic modules were analyzed and described in [15]. The authors highlighted a need of new methods for the wire bond life prediction.

In order to increase joint quality (reliability) some existing solutions could be mentioned. For example, the novel micro-sensor design that uses various piezoresistive stress sensors for measuring localized stresses during copper wire bonding is shown in [16]. Other solution related to the effect reduction of the lift-off failure mechanism allows maintaining mechanically an efficient wire/chip contact through a coating made of polymeric resin on the welding [17].

Some investigations were related to the material migration processes that occur in electrical interconnects during ICs operation. For instance, static EM analysis for on-chip signal interconnects has been analyzed in [18], where authors implemented static analysis approach taking into account different possible switching scenarios as well as proposed methods for modeling multiple simultaneous switching drivers. The EM driven failures on miniature silver fuses of electric cards were observed in [19], where cards are used for cryogenics instrumentation in the Large Hadron Collider accelerator by applying analytical tools and techniques (Scanning Electron Microscopy, spectrometry and Weibull reliability calculations).

Another type of study was performed in terms of the Joule heating effect in the formation of nanogaps by EM [20], where authors evaluated the impact of dissipation during the formation of nanogaps due to EM through the experimentally observed breaking process.

The time distribution of EM failure in small copper interconnects was modeled and observed in [21]. The authors derived approach for the interconnect reliability estimation in terms of investigation of approximate lognormal distribution of EM failure times. Another explanation which relates the normal distribution of activation energies together with the permanence characteristic of the lognormal distribution was presented based on numerical simulations.

The EM multistress pattern technique for copper drift velocity is presented in [22], where authors investigated evolution of drift velocity and its relation with the current density and the temperature by performing package EM tests. The Black's parameters have been defined through the current density investigation too.

Method for the 3D (three-dimensional) modeling of the EM reliability of the interconnects at circuit layout level is presented and broadly explained in [23]. In order to perform such modeling, a 3D finite element circuit model has to be developed from a given two-dimensional IC layout using both Cadence (a circuit simulator) and ANSYS (a finite element software). Once the model is ready, the EM weak 
spots of the interconnects in the IC can be analyzed in terms of the effects of current density, temperature gradient, thermo-mechanical stress gradient on the EM reliability of the ICs.

One of the possible technical solutions to avoid EM problems in local interconnects is the limitation of the length of the wires. However, if the stress is high enough and the dielectrics are weak, metal extrusions may form leading to leakage between neighboring wires [24].

The global increasing tendency of the current densities in the electric power systems has become forcing driver for the consideration of the EM as a critical concern in high-performance designs.

A novel finite-difference based method for power grid EM tracking is described in [25], where authors considered varying voltage and temperature across the die in developed physics-based models. The proposed solution extended the existing physical models for EM in metal branches, where EM degradation in multibranch interconnect trees for on-die power grids has been checked. The authors claim that such method could be applicable for very large power grids.

Another approach for fast and stochastic analysis methodology for EM assessment of power distribution networks is presented in [26], where authors considered novel variations-aware current density model based on Hermite polynomial chaos. The results demonstrated significant margins in EM lifetime when compared with the traditional worst-case approach. The application of the proposed concept was demonstrated in developing some failure criteria of IR drop aware system-level.

The above-presented literature describes extensively the several problems affecting the life-time of the power electronics assemblies (reliability impacts) and some existing solutions are presented too. Most of them devoted to the numerous thermal cycles experienced both in active power cycling and in passive ambient or cooling medium temperature cycling. There are references referring to the impact of the EM phenomenon as well, however only limited number and quite recent studies considered EM in a scale of a high power.

Since EM mechanism remains, but its failure modes and visible evidence have changed, they have to be examined and reconsidered. This study focuses on the EM phenomenon by considering directions of the sparks under a certain arrangement of the connections, which take place depending on the EM denoted to round copper wire at high power (observations under current up to $500 \mathrm{~A}$ ). Such results may be utilized in some future applications and have not been considered by announced researches to best of our knowledge.

The rest of a paper is organized as follows: Section 2 presents the theoretical concept of the conducted theory related to the experimental process. Section 3 shows the conceptual design of the experimental setup. Section 4 reviews testing results of the reported EM phenomena of the joint interconnects. Conclusions are drawn in Section 5.

\section{THEORETICAL BACKGROUND AND EXPERIMENTAL EXPECTATIONS}

In order to predict any device reliability as well as to investigate and better analyze failure modes, some quantitative accelerated tests are used. As it is known, test conditions are an important consideration since the reliability needs to be proceeded for ensuring reliable equipment, its control, and safety of the involved people safety in a testing process. When the accelerated tests are performed, some failure is expected because of humidity, temperature, vibration, electrical current, fatigue, and voltage. Hence high temperature causes some failures, which include erosion, creep, EM and interdiffusion [27]. 
The main focus of the current article is to test the EM at high power. The obtained results will be presented further in Section 4, but before that, let's describe EM basics.

When current flows through a conductor, two forces produced. One is a direct electrostatic force $(\mathrm{Fe})$ which is originated from electric field strength in the copper or aluminium interconnections and has the same direction as the field. Since the positive ions of metals are to somehow shielded by the negative electrons inside the metal, this type of force may be ignored almost in most of cases. Another is often known as the force of electron wind $(\mathrm{Fw})$ caused by the transfer of momentum between electrons forming the conduction and ions of the metal in the crystal lattice. Combining both of the two forces results in causing the ions to move in the direction of electrons (called as current flow). The resulting transport of mass is referred to as EM [3, 28]. The main EM related parameters and definitions are characterized below [28].

The net force (Fnet) on an ion in the metal can be defined as follows (result of the sum of the two forces):

$$
F_{n e t}=F_{e}+F_{w}=\left(N_{e}+N_{w}\right) q E=N^{*}{ }_{e f f} q E
$$

in which $\mathrm{Ne}$ is the number of charges of the ions, $\mathrm{Np}$ is the number of charges corresponding to the electron wind, q denotes the electric charge, $\mathrm{E}$ is the electric field, and $\mathrm{N}^{*}$ eff is the apparent effective number of charges, which is a combination of $\mathrm{Ne}$ and $\mathrm{Nw}$, since the individual contributions cannot be separately measured.

The amount of mass flux that results from the driving forces of EM may be found by using the following relation:

$$
J_{E M}=N \mu F_{n e t}=N_{a}\left(\frac{D_{e f f}}{k_{B} T}\right) N^{*}{ }_{e f f} q \rho j
$$

where JEM stands for the atomic flux caused by EM, Na the atomic density, Deff the total effective diffusivity of the ions of the metal, $\mathrm{kB}$ denotes Boltzmann's constant, $\mathrm{T}$ stands for the temperature (absolute), $\rho$ the electrical resistivity and $\mathrm{j}$ stands for current density.

The main terms, in particular, the electric field (E), the mobility of the metal ions ( $\mu$ ) used in Eq. [2] described as follows:

$$
\begin{gathered}
E=p j \\
\mu=\frac{D_{e f f}}{k_{B} T}
\end{gathered}
$$

The drift velocity (vd) of the ions results from Eq. [2]:

$$
v_{d}=\mu F_{n e t}=\frac{D_{e f f}}{k_{B} T} N^{*}{ }_{e f f} q \rho j
$$

Worth to mention that the values for $\mathrm{N}^{*}$ eff and Deff differ very significantly, and that variation depends on the dominating mechanism of diffusion in the conductor system. There are three types of diffusion; surface diffusion, grain boundary diffusion and bulk diffusion. Grain boundary diffusion is the most 
dominating one in the migration process and exists in aluminum wires, on the other hand surface diffusion is dominating in interconnects made from copper [29].

Based on the above-explained theoretical concept, it is expected that, due to the effect of the momentum of the EM driving forces described in Eq. [2], sparks will follow the situations shown in Fig. 1.

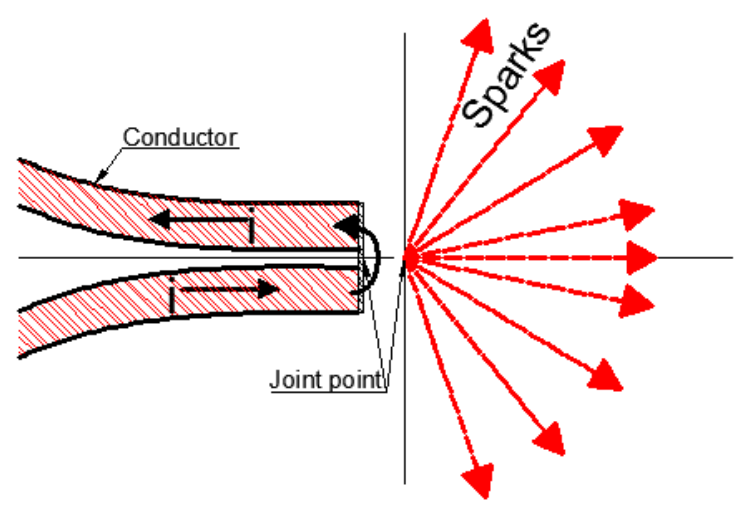

(a)

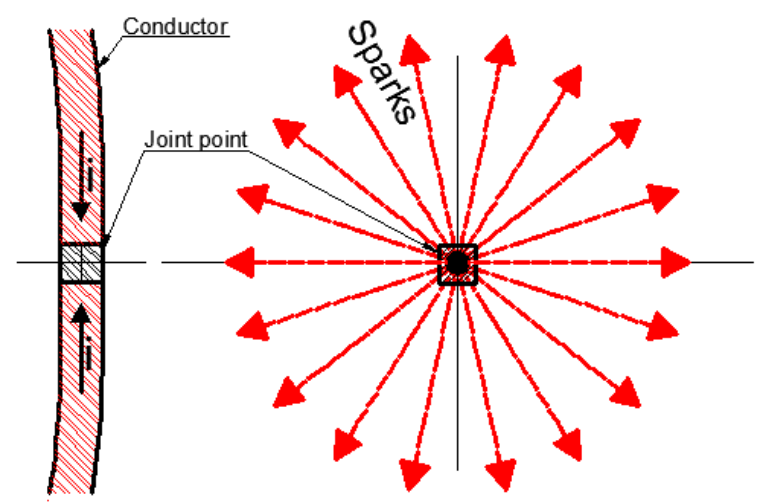

(b)

Figure 1. (a) The first case of the parallel interconnects; (b) The second case of the direct interconnects (dot shows current flow perpendicularly to the plane).

The electrons travel in one direction; in order to reverse the direction of the electrons (their flows' velocity), we need a huge current over a short time period. There are two considered general cases. First, when two conductors are located nearby each other having a sharp corner (a small gap) between them (see Fig.1 a), EM occurs in the weakest point and the sparks' direction exists only in the opposite half plane from the EM observation. In the second case, two round wires connected directly as a joint point, the high probability of a random sparks' directions exists due to the EM (see Fig.1 b).

\section{THEORETICAL CONCEPT OF THE CONDUCTED THEORY}

In order to experimentally satisfy the conceptual design discussed earlier in this study, the schematic diagram shown in Fig.2 (a) was adopted. The switch $\mathrm{S}$ denotes an IGBT by which the ON time interval is controlled. L stands for the inductor which is also shown in Fig.2 (b). It has 350 turns made of copper wire of $1.8 \mathrm{~mm}$ in diameter. The electrical power source in this experiment is achieved by connecting several capacitors in parallel and series to maintain large currents. The total capacitance is $5 \mathrm{mF}$ and can be charged up to $350 \mathrm{~V}$. The weak interconnection shown in the circuit is made intentionally in order to have $100 \%$ probability of having the spark in a specific location if it is going to occur. Simply, the connection is made by putting the ends near to each other as shown in Fig.2 (b). It is noteworthy to mention here that the peaks of injected currents range between $400 \mathrm{~A}$ to $700 \mathrm{~A}$ when the terminal voltage of the capacitors range between $200 \mathrm{~V}$ to $350 \mathrm{~V}$. In addition, since the $\mathrm{ON}$ switching period is relatively small (around 1.5-2.1ms), the chosen cross-sectional area of the wire can afford even larger current than the mentioned ones over this short interval. 


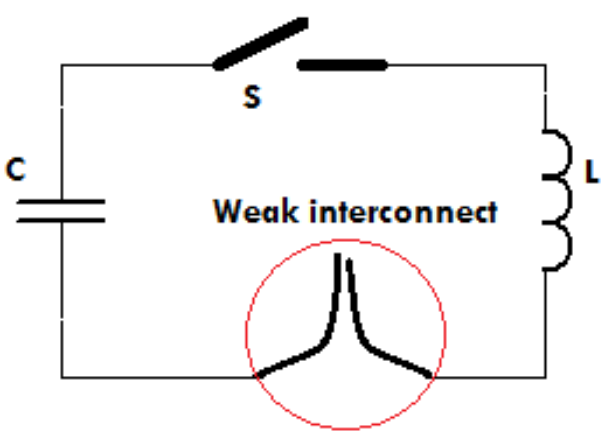

(a)

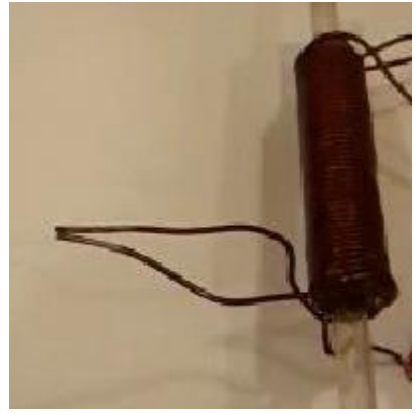

(b)

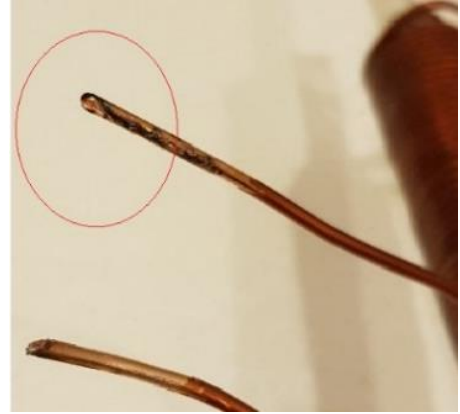

(c)

Figure 2. (a) Schematic diagram of the experimental setup, (b) the weak connection in series with the inductor, (c) scratch in the wire tip

Once the experimental setup is adapted, the practical simulations can be realized.

\section{FINDINGS and DISCUSSION}

It is important to note that the wire connection is made in such a way that the direction of the current is spatially reversed. After generating the spark, a scratch takes place in the weakest connection as shown in Fig.2 (c). Many tests have been performed while connection is made as shown in Fig.2 (b), i.e., the flowing electrons are forced to change their spatial direction.

A slow-motion capturing camera was utilized in order to realize the direction of sparks. In Fig. 3, we present two pictures taken from two different slow-motion videos. In fact, for each of the pictures, three successive shots were merged so that the direction of the sparks is well realized. As seen from Fig. 3, almost all of the sparks are ejected in the half of space whose plane is perpendicular to the direction of current. In other words, due to the electron's momentum direction, the sparks tend to follow that direction as it was expected theoretically and presented in Section 2 (see Fig. 1 (a)).

For sake of having constructive results and as an experimental proof, similar experiments have been also conducted, but this time the interconnect is made in such a way that momentum of moving electrons doesn't change its direction as shown in Fig. 1 (b). In this case, if the sparks occur, they will be in the plane that is perpendicular to the direction of current. Several experiments were performed while having a weak interconnect satisfying the situation in Fig. 1 (b). Fig. 4 present two different experiments. As it can be clearly seen from the figure, the preferred direction of sparks lies within the plane across the connection. 

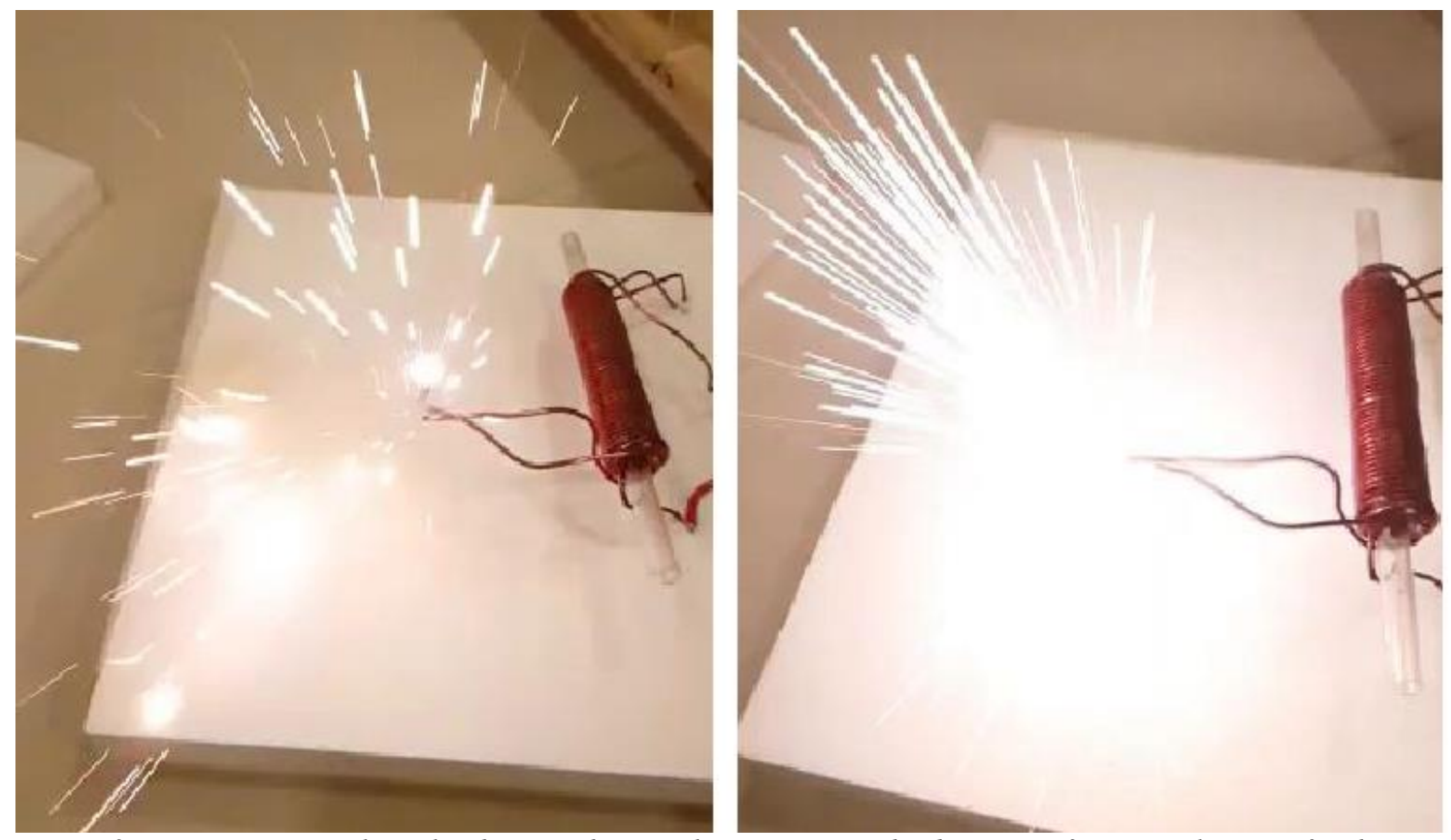

Figure 3. Two experimental results showing that spark ejection is in the direction of moving electrons for the case in Fig. 1 (a)
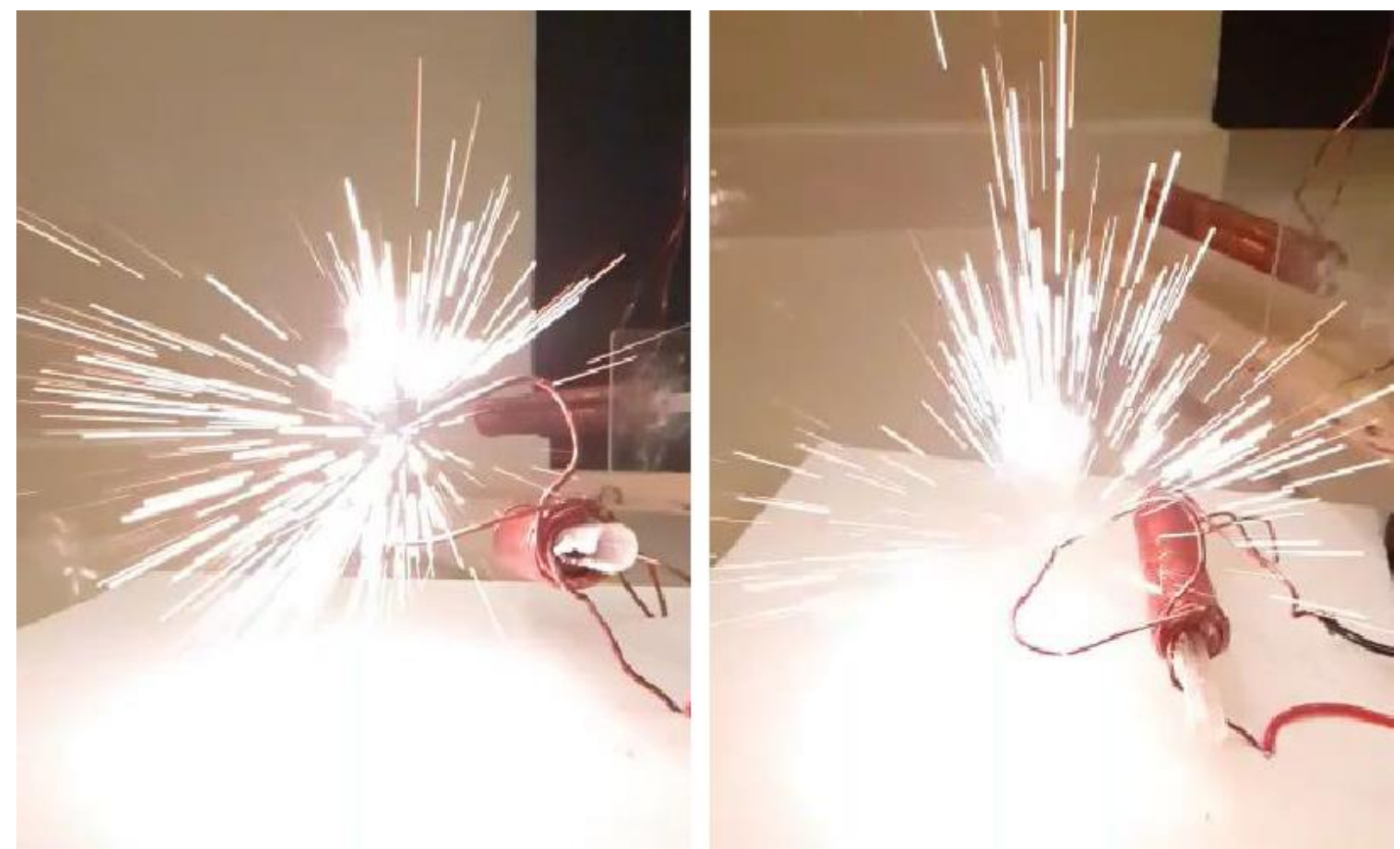

Figure 4. Two experimental results showing the direction of spark ejection for the case in Fig.l (b) 


\section{CONCLUSIONS}

A literature review regarding EM is introduced in the current study. In fact, extensive research has been performed by the authors searching whether any investigation about spark's direction was carried out or not. Therefore, to best of knowledge, the current study presents experimental tests, for the first time, pointing to the fact that sparks follow the spatial direction of currents by considering two particular copper wires' connections - parallel and direct interconnects. In order to realize the effect of the momentum of the electromigration (EM) driving forces in terms of spark's direction, the initial theoretical expectation has been performed for both arrangements of the wires. After that, the experimental setup has been developed with the main purpose of realizing and testing the theoretical expectations for the certain observation concept. The experiments have been conducted at high current (around $500 \mathrm{~A}$ ). As a result, it has been observed that for the first case (parallel connection of the wires) the spark's direction exists only in the opposite half-plane from the EM observation, however for the second case (wires connected as a joint point) - random directions exist as it has been initially expected. Such results are important and have a valuable addition to the field.

There are several phenomena that have affect negatively in some systems, and later on, they were utilized in some other applications. A typical example is the eddy-currents effect but utilized in heat induction. Similarly, the findings in this work may be utilized in nano-technology, for instance. Finally, for future consideration, interesting is to investigate the direction of sparks in relatively much smaller size of conductors and current scales.

\section{REFERENCES}

[1] Black, J. R. Electromigration-A brief survey and some recent results. IEEE Transactions on Electron Devices 1969, 16, 338-347. DOI: 10.1109/T-ED.1969.16754

[2] Ho, P. S. and Kwok, T. Electromigration in metals. Reports on Progress in Physics 1989, 52, 301-348. DOI: $10.1088 / 0034-4885 / 52 / 3 / 002$

[3] Lienig, J, Thiele, M. Fundamentals of Electromigration-Aware Integrated Circuit Design. Fundamentals of Electromigration, Springer International Publishing, Switzerland, 2018.

[4] Canumalla, S, Viswanadham, P. Portable Consumer Electronics: Packaging, Materials, and Reliability. Second level packaging-interconnect technologies, PennWell Corporation, Oklahoma, USA, 2010.

[5] Celnikier, Y, Benabou, L, Dupont, L, Coquery, G. Investigation of the heel crack mechanism in Al connections for power electronics modules. Microelectronics reliability 2011, 51, 965-974. DOI: 10.1016/j.microrel.2011.01.001

[6] Poech, M. H. How materials behaviour affects power electronics reliability. In: $6^{\text {th }}$ International Conference on Integrated Power Electronics Systems, 16-18 March 2010, IEEE, Nuremberg, Germany: pp. 1-6.

[7] Teng, H, Zhang, H, Yang, H, Zhou, M, Tsui, A. C. Effect of moisture and temperature on Al-Cu interfacial strength. In: International Conference on Electronic Packaging Technology \& High Density Packaging, 2831 July 2008, IEEE, Shanghai, China: pp. 1-4.

[8] Onuki, J, Koizumi, M, Araki, I. Investigation of the Reliability of Copper Ball Bonds to Aluminum Electrodes. IEEE Transactions on components, hybrids, and manufacturing technology 1987, 10, 550-555. DOI: 10.1109/TCHMT.1987.1134799

[9] Tian, Y, Hang, C, Wang, C, Zhou, Y. Evolution of Cu/Al Intermetallic Compounds in the Copper Bump bonds during Aging Process. In: $8^{\text {th }}$ International Conference on Electronic Packaging Technology, 14-17 Aug. 2007, IEEE, Shanghai, China: pp. 1-5.

[10] Bashir, M. N., Haseeb, A.S.M.A., Rahman, A.Z.M.S., et al. Reduction of electromigration damage in SAC305 solder joints by adding Ni nanoparticles through flux doping. Journal of Materials Science 2015, 50, pp. 67486756. DOI: https://doi.org/10.1007/s10853-015-9230-7

[11] Smeby, J. Solder Joint Behavior in HCC/PWB Interconnections. IEEE Transactions on Components, Hybrids, and Manufacturing Technology 1985, 8, 391-396. DOI: 10.1109/TCHMT.1985.1136512 
[12] Takahashi, T, Yu, Q. Precision evaluation for thermal fatigue life of power module using coupled electricalthermal-mechanical analysis. In: 12 th Electronics Packaging Technology Conference, 8-10 Dec. 2010, IEEE, Singapore, Singapore: pp. 201-205.

[13] Anzawa, T, Yu, Q, Yamagiwa, M, Shibutani, T, Shiratori, M. Power cycle fatigue reliability evaluation for power device using coupled electrical-thermal-mechanical analysis. In: $11^{\text {th }}$ Intersociety Conference on Thermal and Thermomechanical Phenomena in Electronic Systems, 28-31 May 2008, IEEE, Orlando, Florida, USA: pp. 815-821.

[14] Anzawa, T, Yu, Q, Shibutani, T, Shiratori, M. Reliability Evaluation for Power Electronics Device using Electrical Thermal and Mechanical Analysis. In: $9^{\text {th }}$ Electronics Packaging Technology Conference, 10-12 Dec. 2007, IEEE, Singapore, Singapore: pp. 94-99.

[15] Yang, L, Agyakwa, P. A, Johnson, and C. M. Physics-of-Failure Lifetime Prediction Models for Wire Bond Interconnects in Power Electronic Modules. IEEE Transactions on Device and Materials Reliability 2013, 13, 9-17. DOI: 10.1109/TDMR.2012.2235836

[16] Zhang, X, Selvanayagam, C. S, Yong, W. Y, Chai, T. C, Trigg, A. D. Design and development of microsensors for measuring localised stresses during copper wirebonding. In: $14^{\text {th }}$ Electronics Packaging Technology Conference (EPTC), 5-7 Dec. 2012, IEEE, Singapore, Singapore: pp. 244-250.

[17] Hamidi A, Team, P.P. Introduction to semiconductor packaging in high power electronics. Seminaire ECPE, May 2004.

[18] Blaauw, D. T., Chanhee Oh, Zolotov, V, Dasgupta, A. Static electromigration analysis for on-chip signal interconnects. IEEE Transactions on Computer-Aided Design of Integrated Circuits and Systems 2003, 22, 39-48. DOI: 10.1109/TCAD.2002.805728

[19] Trikoupis, N, Casas, J, Fontenla, A.T.P. Electromigration driven failures on miniature silver fuses at the Large Hadron Collider. In: Proceedings of Science of Topical Workshop on Electronics for Particle Physics (TWEPP-17), 11-15 Sep. 2017, Santa Cruz, California, USA: pp. 066.

[20] Trouwborst, M.L, Molen, S.J, Wees, B.J. The role of Joule heating in the formation of nanogaps by electromigration. Journal of Applied Physics 2006, 99, 1-9. DOI: 10.1063/1.2203410

[21] Dwyer, V.M. Modeling the electromigration failure time distribution in short copper interconnects. Journal of Applied Physics 2008, 104, 1-12. DOI: 10.1063/1.2970171

[22] Doyen, L, Federspiel, X, Arnaud, L, Terrier, F, Wouters, Y, Girault, V. Electromigration multistress pattern technique for copper drift velocity and Black's parameters extraction. In: International Integrated Reliability Workshop Final Report, 15-18 Oct. 2007, IEEE, S. Lake Tahoe, Canada, USA: pp. 74-78.

[23] Tan C.M., He F., Electromigration Modeling at Circuit Layout Level. Springer Briefs in Applied Sciences and Technology. Springer, Singapore, 2013.

[24] Gambino J., Process technology for copper interconnects. Handbook of Thin Film Deposition. Techniques, Processes, and Technologies (3 ${ }^{\text {rd }}$ Edition), William Andrew Publishing, Waltham, USA, 2012.

[25] Chatterjee, S, Sukharev, V, Najm, F. N. Power Grid Electromigration Checking Using Physics-Based Models. IEEE Transactions on Computer-Aided Design of Integrated Circuits and Systems 2018, 37, 1317-1330. DOI: 10.1109/TCAD.2017.2666723

[26] Jain, P, Mishra, V, Sapatnekar, S. S. Fast Stochastic Analysis of Electromigration in Power Distribution Networks. IEEE Transactions on Very Large Scale Integration (VLSI) Systems 2017, 25, 2512-2524. DOI: 10.1109/TVLSI.2017.2706520

[27] Calixto, E. Accelerated life test, reliability growth analysis, and probabilistic degradation analysis. Gas and Oil Reliability Engineering: Modeling and Analysis ( $2^{\text {nd }}$ Edition). Gulf Professional Publishing, Texas, USA, 2016.

[28] Meyer, M. A. Effects of advanced process approaches on electromigration degradation of Cu on-chip interconnects, $\mathrm{PhD}$, Brandenburg University of Technology Cottbus-Senftenberg, Germany, 2007.

[29] Lienig, J. Interconnect and current density stress - an introduction to electromigration-aware design. In : Proceedings of the SLIP '05 International workshop on System level interconnect prediction, 2-3 April 2005, San Francisco, California, USA : pp. 81-88. 